\title{
Gate-All-Around Single-Crystal-Like Poly-Si Nanowire TFTs With a Steep-Subthreshold Slope
}

\author{
Tung-Yu Liu, Shen-Chuan Lo, and Jeng-Tzong Sheu
}

\begin{abstract}
We investigate the characteristics of single-crystallike (SCL) poly-Si nanowire (SCL poly-Si NW) thin-filmtransistors with gate-all-around (GAA) structures. The GAA SCL poly-Si NWs are prepared by a modified sidewall spacer process utilizing an amorphous silicon $(\alpha-\mathrm{Si})$ mesa structure. The combination of the high surface-to-volume ratio of the NW and a nominal gate length of $0.25 \mu \mathrm{m}$ lead to clear improvement in electrical performance, including a steep subthreshold swing $(90 \pm 15 \mathrm{mV} / \mathrm{dec})$, a virtual absence of drain-induced barrier lowering $(21 \pm 13 \mathrm{mV} / \mathrm{V})$, and a very high $\mathrm{ON} / \mathrm{OFF}$ current ratio $\sim 7 \times 10^{7}\left(\mathrm{~V}_{\mathrm{D}}=1 \mathrm{~V}, \mathrm{~V}_{\mathrm{G}}=3 \mathrm{~V}\right)$.
\end{abstract}

Index Terms-Gate-all-around (GAA), nanowire (NW), single-crystal-like (SCL), thin film transistor (TFT).

\section{INTRODUCTION}

D UE TO the simplicity in device preparation and excellent performance in electrical properties, polycrystalline silicon thin-film transistors (Poly-Si TFTs) have been studied and applied in many applications, such as active matrix liquid crystal displays [1] and memory devices [2]. Recently, poly-Si TFTs utilizing nanowire (NW) channel with multiplegate structures have been demonstrated to meet demands in both TFT performance and suppression of short channel effects encountered during scale-down [3]-[5]. Although gate controllability was enhanced through multiple-gate structures, defects at grain boundaries and dangling bonds in the poly-Si NW channel undermine the carrier mobility and the device switching speed [6]. Different techniques such as solid-phase crystallization (SPC) [7], metal-induced-lateral crystallization [8], and excimer laser annealing [9] have been proposed to increase the grain size so that trap-state density can be reduced. Plasma treatment has also been demonstrated to reduce the defect density [5], [10]. In addition, reduction of defect density and improvement of gate controllability via scale-down of the poly-Si NW channel have been reported. Some studies have even demonstrated the scale-down of the poly-Si NW without using advanced lithographic tools and have obtained excellent device characteristics [11]-[14]. Poly-Si NW TFTs with small gate length $(<0.1 \mu \mathrm{m})$ and very thin dielectrics have also been proposed to improved transfer characteristics

Manuscript received October 4, 2012; accepted February 12, 2013. Date of publication March 7, 2013; date of current version March 20, 2013. The review of this letter was arranged by Editor K. Uchida.

T.-Y. Liu and J.-T. Sheu are with the Department of Materials Science and Engineering, Institute of Nanotechnology, National Chiao Tung University, Hsinchu 30050, Taiwan (e-mail: jtsheu@faculty.nctu.edu.tw).

S.-C. Lo is with the Material and Chemical Research Laboratories, Industrial Technology Research Institute, Hsinchu 31040, Taiwan.

Color versions of one or more of the figures in this letter are available online at http://ieeexplore.ieee.org.

Digital Object Identifier 10.1109/LED.2013.2247737

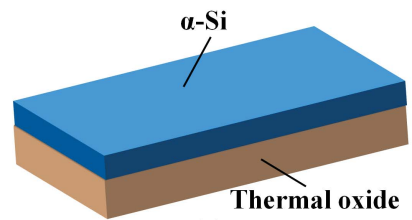

(a)

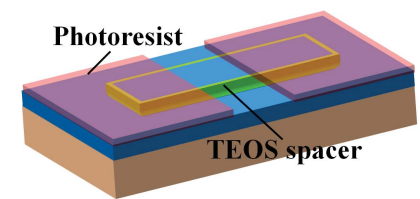

(c)

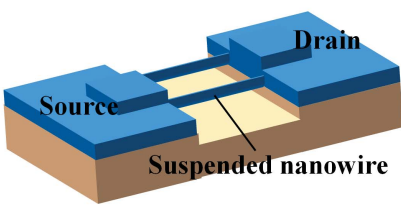

(e)

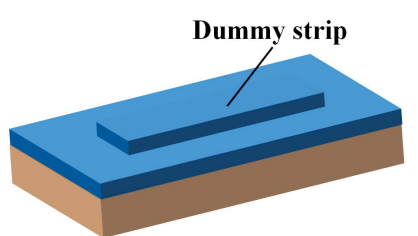

(b)

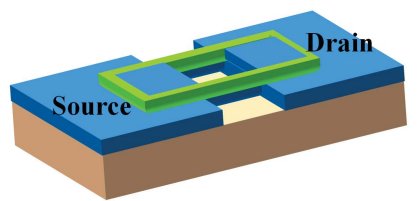

(d)

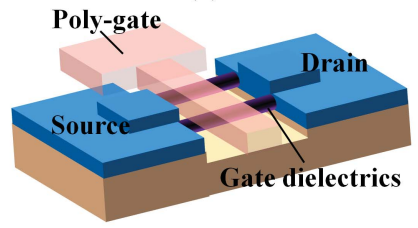

(f)
Fig. 1. (a)-(f) Key process flow of device fabrication using the simplified sidewall spacer technique.

[15], [16]. However, short-channel effects (SCEs) like large leakage current and drain-induced barrier lowering (DIBL) have emerged as problems. Therefore, poly-Si TFT with the GAA structure still suffers from intrinsic defects in the polySi channel. In this letter, we present a novel modified sidewall spacer process to prepare poly-Si NW TFTs with a gateall-around (GAA) structure. The nanowire channel exhibits SCL structure after SPC process. Without any trap-reduced plasma treatment, the GAA SCL poly-Si NW TFTs possessing channels of high surface-to-volume ratio and a $0.25-\mu \mathrm{m}$ gate length exhibited superior channel controllability and the ability to suppress SCEs.

\section{DEVICE FABRICATION}

The GAA SCL poly-Si NW TFTs were fabricated by a simplified top-down sidewall spacer technique, with the key process flow shown in Fig.1. Wafers with 500-nm thick thermal oxide were grown as the starting substrate layer, and a $70-\mathrm{nm}$ thick amorphous silicon $(\alpha-\mathrm{Si})$ layer was deposited using low pressure chemical vapor deposition (LPCVD) at $550{ }^{\circ} \mathrm{C}$ [Fig. 1(a)]. Next, the $\alpha$-Si layer was patterned into a mesa structure through optical lithography, which was followed by reactive ion etching (RIE). The $\alpha$-Si layer was transformed into two parts; the thicker part served as a 


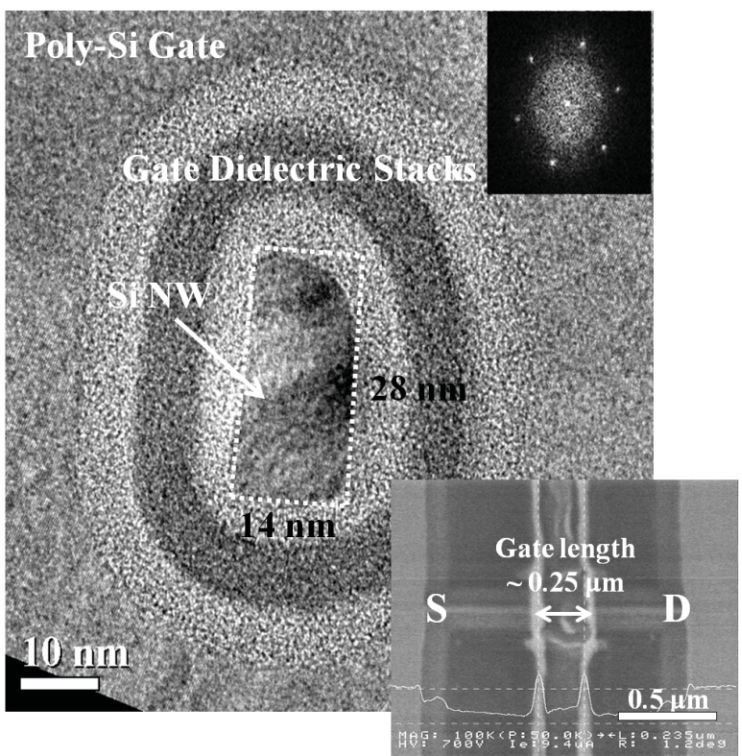

Fig. 2. Cross-section TEM image of a GAA SCL poly-Si NW TFT. The $\mathrm{NW}$ was surrounded by gate dielectric stacks $(\mathrm{ONO}=5 \mathrm{~nm} / 8 \mathrm{~nm} / 8 \mathrm{~nm})$ and a poly-Si gate. Upper inset: FFT patterns confirming the zone axis of the $\mathrm{Si}$ NWs is in the [110] direction. Lower inset: SEM image confirming the gate length of the GAA SCL poly-Si NW TFT is around $0.25 \mu \mathrm{m}$.

dummy strip, and the thinner one served as device active layer [Fig. 1(b)]. A 60-nm thick tetraethylorthosilicate (TEOS) layer was deposited using LPCVD, and then etched through RIE, leaving TEOS spacers. Before NW formation, the source and drain $(\mathrm{S} / \mathrm{D})$ regions were defined through an I-line stepper [Fig. 1(c)]. Next, the $\alpha$-Si layer was etched through highselective RIE, and the NWs were formed through TEOS hard masks [Fig. 1(d)]. After NWs formation, the TEOS hard masks were removed in 1:50 diluted HF solution. Next, the SPC was performed at $600{ }^{\circ} \mathrm{C}$ for $24 \mathrm{~h}$ in nitrogen ambient to turn the $\alpha$-Si into polycrystalline silicon. Then the NWs were released from the bottom thermal oxide during the RCA cleaning [Fig. 1(e)]. The TEOS oxide/ nitride/ TEOS oxide $(\mathrm{O} / \mathrm{N} / \mathrm{O}=$ $5 \mathrm{~nm} / 8 \mathrm{~nm} / 8 \mathrm{~nm}$ ) gate dielectric stacks and a 200-nm in situ $\mathrm{N}^{+}$doped poly-Si film were placed surrounding NWs by using LPCVD conformal deposition. After the patterning of the poly-Si gate [Fig. 1(f)], the wafers were doped with phosphorus for the S/D at a dose of $5 \times 10^{15} \mathrm{~cm}^{-2}(10 \mathrm{keV})$ through self-aligned implantation to reduce the contact resistance and the series resistance of the S/D access regions. A 300-nm thick TEOS layer was deposited as the passivation layer using LPCVD. Then, samples were activated at $600{ }^{\circ} \mathrm{C}$ for $8 \mathrm{~h}$ in nitrogen ambient. The contact holes were defined, and $\mathrm{Al}$ metallization was performed. Finally, the wafers were sintered at $450{ }^{\circ} \mathrm{C}$ in a mixture of hydrogen $(5 \%)$ and nitrogen (95\%) ambient for $30 \mathrm{~min}$. Despite the fact that the O/N/O gate stack undermines gate controllability, this poly-Si NW TFT device with surrounding ONO layers were prepared for potential memory applications.

\section{RESULTS AND DISCUSSION}

Fig. 2 presents the high-resolution TEM channel crosssection image of a GAA SCL poly-Si NW TFT. The NW

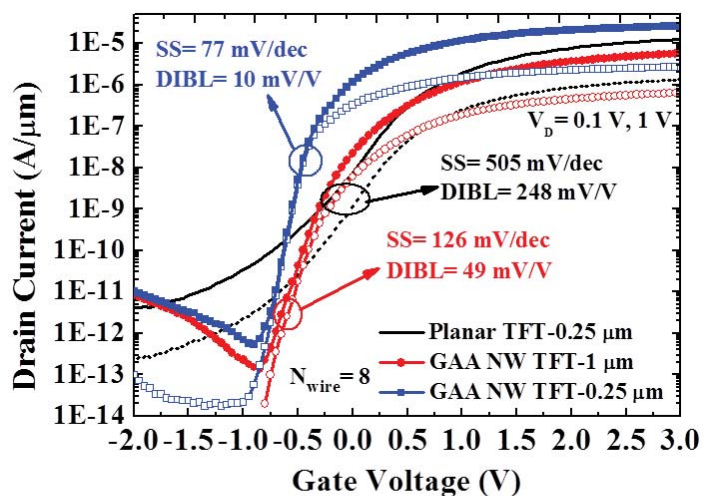

(a)

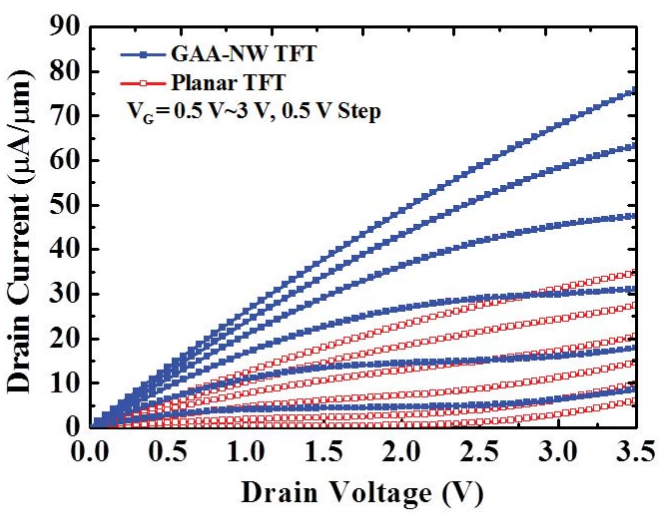

(b)

Fig. 3. (a) Transfer characteristics for a GAA SCL poly-Si NW TFT (gate lengths of $0.25 \mu \mathrm{m}$ and $1 \mu \mathrm{m}$ ) and conventional planar TFT. (b) Comparison of output characteristics between the GAA SCL poly-Si NW TFT and conventional planar TFT.

channel is surrounded by ONO stacks (TEOS/nitride/TEOS = $5 \mathrm{~nm} / 8 \mathrm{~nm} / 8 \mathrm{~nm}$ ) and a poly-Si gate with channel width $\sim 14$ $\mathrm{nm}$ and thickness $\sim 28 \mathrm{~nm}$. The upper inset is fast Fourier transform (FFT) patterns confirming that the zone axis of the Si NWs is the [110] direction. The FFT pattern also shows that the Si NWs are highly crystalline. The lower inset SEM image confirms that the gate length of GAA SCL poly-Si NW TFT is $\sim 0.25 \mu \mathrm{m}$. In the process flow, the TEOS oxide was used as a sidewall hard mask instead of traditional silicon nitride hard mask. Without using the time-consuming wet etching process to remove silicon nitride and the additional dummy layer material, GAA SCL poly-Si NWs can be prepared efficiently with an improved device yield.

Fig. 3(a) presents a comparison of the normalized transfer characteristics $\left(\mathrm{I}_{\mathrm{D}}-\mathrm{V}_{\mathrm{G}}\right.$ curve) between the GAA SCL poly-Si NW TFT and a planar TFT without any plasma-related treatment. The gate lengths of the TFTs were $0.25 \mu \mathrm{m}$, and the effective width of the GAA SCL poly-Si NW and planar TFTs were $0.672 \mu \mathrm{m}(84 \mathrm{~nm} \times 8)$ and $1.1 \mu \mathrm{m}$, respectively. The GAA SCL poly-Si NW TFT exhibits a threshold voltage of $\sim-0.4 \mathrm{~V}$ at $\mathrm{I}_{\mathrm{D}}=\left(\mathrm{W}_{\mathrm{eff}} / \mathrm{L}\right) \times 10^{-8} \mathrm{~A}$ and $\mathrm{V}_{\mathrm{D}}=0.1 \mathrm{~V}$. An analysis of 24 TFTs showed that the GAA SCL poly-Si NW TFT exhibits a very small subthreshold swing $(90 \pm 15$ $\mathrm{mV} / \mathrm{dec}$, extracted from $\mathrm{I}_{\mathrm{D}}=10^{-8} \mathrm{~A}$ to $\mathrm{I}_{\mathrm{D}}=10^{-12} \mathrm{~A}$ and $\mathrm{V}_{\mathrm{D}}=0.1 \mathrm{~V}$ ), one which is superior to that of the planar 
TABLE I

Characteristics of ReCEnTLy RePorted POLY-Si NW TFTS USING SPC TECHNIQUE

\begin{tabular}{|c|c|c|c|c|c|}
\hline Source & $\begin{array}{l}\text { This } \\
\text { Letter }\end{array}$ & [5] & [14] & [15] & [16] \\
\hline $\begin{array}{c}\text { Gate } \\
\text { structure }\end{array}$ & GAA & GAA & $\begin{array}{c}\text { Double- } \\
\text { gated }\end{array}$ & $\Omega$-gate & $\Omega$-gate \\
\hline $\begin{array}{c}\text { Gate length } \\
(\mu \mathbf{m})\end{array}$ & 0.25 & 2 & 1 & 0.1 & 0.09 \\
\hline $\begin{array}{c}\text { Channel } \\
\text { dimension } \\
\left(\mathbf{n m}^{2}\right)\end{array}$ & $14 \times 28$ & $75 \times 38$ & $18 \times 72$ & $14 \times 17$ & $20 \times 10$ \\
\hline $\begin{array}{c}\mathrm{NH}_{3} \text { plasma } \\
\text { treatment }\end{array}$ & $\mathrm{W} / \mathrm{O}$ & $1 \mathrm{~h}$ & $3 \mathrm{~h}$ & $\mathrm{~W} / \mathrm{O}$ & $\mathrm{W} / \mathrm{O}$ \\
\hline EOT (nm) & 18 & 20 & 20 & 1.7 & $\begin{array}{c}10 \\
\left(\mathrm{HfO}_{2}\right)\end{array}$ \\
\hline S.S. (mV/dec) & $90 \pm 15$ & 114 & 115 & 79 & 113 \\
\hline DIBL $(\mathrm{mV} / \mathrm{V})$ & $21 \pm 13$ & 13 & 60 & 78 & 251 \\
\hline $\begin{array}{c}\mathbf{I}_{\text {ON }} / I_{\text {OFF }} \\
\mathbf{V}_{\mathbf{G}} ; \mathbf{V}_{\mathbf{D}}\end{array}$ & $\begin{array}{l}>>10^{7} \\
3 \mathrm{~V} ; 1 \mathrm{~V}\end{array}$ & $\begin{array}{c}>10^{8} \\
6 \mathrm{~V} ; 3 \mathrm{~V}\end{array}$ & $\begin{array}{l}>10^{7} \\
6 \mathrm{~V} ; 3 \mathrm{~V}\end{array}$ & $\begin{array}{l}>10^{4} \\
1 \mathrm{~V} ; 1 \mathrm{~V}\end{array}$ & $\begin{array}{l}>10^{7} \\
6 \mathrm{~V} ; 1 \mathrm{~V}\end{array}$ \\
\hline
\end{tabular}

device $(396 \pm 57 \mathrm{mV} / \mathrm{dec})$. Furthermore, the DIBL of the GAA SCL poly-Si NW and planar TFTs are $21 \pm 13 \mathrm{mV} / \mathrm{V}$ and $237 \pm 97 \mathrm{mV} / \mathrm{V}$ (extracted from $\Delta \mathrm{V}_{\mathrm{gs}} / \Delta \mathrm{V}_{\mathrm{ds}}$ of $\mathrm{V}_{\mathrm{D}}=$ 0.1 and $1 \mathrm{~V}$ at $\mathrm{I}_{\mathrm{D}}=10^{-10} \mathrm{~A}$ ), respectively. Furthermore, we suspect that large variations in both SS and DIBL could be further improved by increasing the grain size under the gate. The $0.25-\mu \mathrm{m}$ planar device exhibits SCEs, including a large SS and a clear DIBL due to inferior gate controllability. However, the $0.25-\mu \mathrm{m}$ GAA poly-Si NW TFT shows gateinduced leakage current (GIDL) in the OFF state as the result of stronger electric field between the gate and drain. The transfer characteristics of a 1- $\mu \mathrm{m}$ GAA poly-Si NW TFT are also shown in Fig. 3(a). Despite the $1-\mu \mathrm{m}$ device exhibiting a lower leakage current, the $0.25-\mu \mathrm{m}$ device exhibits superior transfer characteristics owing to the short gate length and the reduction of grain boundaries under the gate area. We suspect that the gate length between $0.1 \mu \mathrm{m}$ and $0.25 \mu \mathrm{m}$ may be an appropriate length for further improvement of device characteristics. Fig. 3(b) presents a comparison of output characteristics between GAA SCL poly-Si NW and conventional planar TFTs. The proposed NW device exhibits a saturation current improvement of $223 \%$ at $\mathrm{V}_{\mathrm{G}}=3 \mathrm{~V}$ and $\mathrm{V}_{\mathrm{D}}=3.5 \mathrm{~V}$ (NW TFT $\sim 76 \mu \mathrm{A} / \mu \mathrm{m}$ over the planar device $\sim 34 \mu \mathrm{A} / \mu \mathrm{m})$. Table I shows a performance summary for the recently reported poly-Si NW TFTs using the SPC technique [5], [14]-[16]. Poly-Si NW TFTs with low SS and high ON/OFF current ratio were most usually subjected to $\mathrm{NH}_{3}$ plasma treatment. In the absence of a lightly doped drain, devices with a gate length smaller than $0.1 \mu \mathrm{m}$ usually suffered from SCEs. However, this GAA SCL poly-Si NW TFT with a $0.25-\mu \mathrm{m}$ gate length and a very small NW channel $\left(14 \times 28 \mathrm{~nm}^{2}\right)$ exhibited single-crystal like electrical properties.

\section{CONCLUSION}

We characterized GAA SCL poly-Si NW TFTs prepared by a novel modified side-wall spacer technique. Without the need for plasma treatment, the GAA SCL poly-Si NW TFTs showed an excellent S.S. $(90 \pm 15 \mathrm{mV} / \mathrm{dec})$, a very small DIBL $(21 \pm 13 \mathrm{mV} / \mathrm{V})$, and a high ON/OFF current ratio $\sim 7 \times 10^{7}$ at a relatively low voltage condition $\left(\mathrm{V}_{\mathrm{G}}=3 \mathrm{~V}\right.$ and $V_{D}=1 \mathrm{~V}$ ), resulting from the very small channel and the reduction of grain boundaries and defects under the gate area.

\section{ACKNOWLEDGMENT}

The authors would like to thank MOE-ATU and NSC, Taiwan for financial support and National Nano Device Laboratories for facility utilization.

\section{REFERENCES}

[1] S. M. Fluxrnan, "Design and performance of digital polysilicon thinfilm-transistor circuits on glass," IEEE Proc. Circuits Devices Syst., vol. 141, no. 1, pp. 56-59, Feb. 1994.

[2] M. Rodder and S. Aur, "Utilization of plasma hydrogenation in stacked SRAM's with poly-Si PMOSFET's and bulk-Si NMOSFET's," IEEE Electron Device Lett., vol. 12, no. 5, pp. 233-235, May 1991.

[3] S. Zhang, R. Han, J. K. O. Sin, and M. Chan, "A novel self-aligned double-gate TFT technology," IEEE Electron Device Lett., vol. 22, no. 11, pp. 530-532, Nov. 2001.

[4] Y.-C. Wu, P.-W. Su, C.-W. Chang, and M.-F. Hung, "Novel twin polySi thin film transistors EEPROM with trigate nanowire structure," IEEE Electron Device Lett., vol. 29, no. 11, pp. 1226-1228, Nov. 2008.

[5] J.-T. Sheu, P.-C. Huang, T.-S. Sheu, C.-C. Chen, and L.-A. Chen, "Characteristics of gate-all-around twin poly-Si nanowire thin-film transistors," IEEE Electron Device Lett., vol. 30, no. 2, pp. 139-141, Feb. 2009.

[6] T.-F. Chen, C.-F. Yeh, and J.-C. Lou, "Effect of grain boundaries on performance and hot-carrier reliability of excimer-laser annealed polycrystalline silicon thin film transistors," J. Appl. Phys., vol. 95, no. 10, pp. 5788-5794, May 2004.

[7] M. K. Hatalis and D. W. Greve, "Large grain polycrystalline silicon by low-temperature annealing of low pressure chemical vapor deposited amorphous silicon films," J. Appl. Phys., vol. 63, no. 7, pp. 2260-2266, Apr. 1988.

[8] S.-W. Lee and S.-K. Joo, "Low temperature poly-Si film transistor fabrication by metal induce lateral crystallization," IEEE Electron Device Lett., vol. 17, no. 4, pp. 160-162, Apr. 1996.

[9] C.-W. Lin, L.-J. Cheng, Y.-L. Lu, Y.-S. Lee, and H.-C. Cheng, "Highperformance low-temperature poly-Si TFTs crystallized by excimer laser irradiation with recessed-channel structure," IEEE Electron Device Lett., vol. 22, no. 6, pp. 269-271, Jun. 2001.

[10] H.-C. Cheng, F.-S. Wang, and C.-Y. Huang, "Effects of $\mathrm{NH}_{3}$ plasma passivation on N-channel polycrystalline silicon thin-film transistors," IEEE Electron Device Lett., vol. 44, no. 1, pp. 64-66, Jan. 1997.

[11] H.-C. Lin, H.-H. Hsu, C.-J. Su, and T.-Y. Huang, "A novel multiplegate polycrystalline silicon nanowire transistor featuring an inverseT gate," IEEE Electron Device Lett., vol. 29, no. 7, pp. 718-720, Jul. 2008.

[12] C.-J. Su, T.-I. Tsai, Y.-L. Liou, Z.-M. Lin, H.-C. Lin, and T.-S. Chao, "Gate-all-around junctionless transistors with heavily doped polysilicon nanowire channels," IEEE Electron Device Lett., vol. 32, no. 4, pp. 521-523, Apr. 2011.

[13] T.-C. Liao, S.-W. Tu, M.-H. Yu, W.-K. Lin, C.-C. Liu, K.-J. Chang, Y.-H. Tai, and H.-C. Cheng, "Novel gate-all-around poly-Si TFTs with multiple nanowire channels," IEEE Electron Device Lett., vol. 29, no. 8, pp. 889-891, Aug. 2008.

[14] W.-C. Chen, C.-D. Lin, H.-C. Lin, and T.-Y. Huang, "A Novel doublegated nanowire TFT and investigation of its size dependency," in Proc. Int. Symp. VLSI Technol. Syst. Appl., Apr. 2009, pp. 121-122.

[15] M. Im, J.-W. Han, H. Lee, L.-E. Yu, S. Kim, C.-H. Kim, S. C. Jeon, K. H. Kim, G. S. Lee, J. S. Oh, Y. C. Park, H. M. Lee, and Y.K. Choi, "Multiple-gate CMOS thin-film transistor with polysilicon nanowire," IEEE Electron Device Lett., vol. 29, no. 1, pp. 102-105, Jan. 2008.

[16] C.-M. Lee and B.-Y. Tsui, "High-performance poly-Si nanowire thinfilm transistors using the $\mathrm{HfO}_{2}$ gate dielectric," IEEE Electron Device Lett., vol. 32, no. 3, pp. 327-329, Mar. 2011. 